\title{
Danos à saúde e qualidade de vida no trabalho de enfermeiros hospitalares: um estudo transversal
}

\author{
Hospital nurses' health and quality of life at work harms: a cross-sectional study \\ Daños a la salud y calidad de vida en el trabajo de las enfermeras hospitalarias: un estudio transversal
}

\author{
Anna Bianca Ribeiro Melo'; Janaina Moreno de Siqueiral"; Marize Barbosa Silvall'; \\ Pablo Alexandre Silva ${ }^{\prime V}$; Glória Maria de Moraes Antonian ${ }^{v}$; Sheila Nascimento Pereira de Farias ${ }^{V I}$
}

\begin{abstract}
RESUMO
Objetivo: analisar a associação entre danos à saúde e qualidade de vida no trabalho de enfermeiros hospitalares. Método: estudo quantitativo, descritivo, transversal, com.145 enfermeiros, aprovado pela comissão de ética sob protocolos $1.634 .051 \mathrm{e}$ 1.643.912. Utilizaram-se estatísticas descritivas, análises bivariadas, teste qui-quadrado, cálculo da razão de chance e intervalos de confiança. Resultados: observou-se que o consumo de medicamentos provocado/agravado pelo trabalho aumentou em 2,31 vezes a chance de o enfermeiro ter baixa qualidade de vida no trabalho; transtornos do sono provocados/agravados pelo trabalho aumentou em 3,15 vezes a chance de ter baixa qualidade de vida no trabalho; cefaleia frequente provocada/agravada pelo trabalho aumentou a chance em 1,98 vezes de ter baixa qualidade de vida no trabalho. Conclusão: condições de trabalho impactam na saúde e qualidade de vida do trabalho do enfermeiro. Ambientes de trabalho adequados proporcionam satisfação pessoal e profissional, além de manterem a qualidade da força de trabalho do enfermeiro.
\end{abstract}

Descritores: Enfermagem; Doença; Qualidade de Vida; Condições de Trabalho; Saúde do Trabalhador.

\begin{abstract}
Objective: to examine the association between health harm and quality of life in the work of hospital nurses. Method: this quantitative, descriptive, cross-sectional study, with 145 nurses, after approval by research ethics committee - protolcols 1.634.051 and 1.643.912. Descriptive statistics, bivariate analyses, chi-square test, odds ratio and confidence intervals were used. Results: the odds of nurses' quality of life at work being poor were found to be increased 2.31 times by work-related or aggravated medicine consumption; 3.15 times by work-related or -aggravated sleep disorders; and 1.98 times by frequent workrelated or -aggravated headache. Conclusion: working conditions impact on nurses' health and quality of life at work. Appropriate work environments favor personal and professional satisfaction and maintain the quality of the nurse's workforce. Descriptors: Nursing; Disease; Quality of Life; Working Conditions; Occupational Health.
\end{abstract}

\section{RESUMEN}

Objetivo: examinar la asociación entre daños a la salud y calidad de vida en el trabajo de enfermeras hospitalarias. Método: estudio cuantitativo, descriptivo, transversal, con 145 enfermeros, previa aprobación del comité de ética en investigación protolcols 1.634 .051 y 1.643.912. Se utilizó estadística descriptiva, análisis bivariados, prueba de chi-cuadrado, razón de probabilidades e intervalos de confianza. Resultados: se encontró que las probabilidades de que la calidad de vida de las enfermeras en el trabajo sea deficiente aumentaban 2,31 veces por el consumo de medicamentos relacionados con el trabajo o agravados; 3,15 veces por trastornos del sueño relacionados con el trabajo o agravados; y 1,98 veces por dolor de cabeza frecuente relacionado con el trabajo o agravado. Conclusión: las condiciones laborales repercuten en la salud y la calidad de vida de las enfermeras en el trabajo. Los entornos laborales adecuados favorecen la satisfacción personal y profesional y mantienen la calidad de la fuerza laboral de la enfermería.

Descriptores: Enfermería; Enfermidad; Calidad de Vida; Condiciones de Trabajo; Salud Laboral.

\section{INTRODUÇÃo}

Entende-se por condições de trabalho os fatores físicos, sociais e administrativos relativos ao meio no qual um trabalhador exerce a sua atividade profissional. Conscientes da importância de estudar as condições de trabalho da enfermagem e identificar a precária situação desses trabalhadores, a Organização Internacional do Trabalho (OIT) e a Organização Mundial da Saúde (OMS) vêm estudando a profissão de enfermeiro ${ }^{1}$.

'Enfermeira. Doutora. Professora Adjunta. Faculdade de Medicina de Petrópolis, Rio de Janeiro, Brasil. E-mail: annabmelo@yahoo.com.br. ORCID: http://orcid.org/0000-0001-8921-2250 "Enfermeira. Mestranda. Universidade Federal do Rio de Janeiro, Rio de Janeiro, Brasil. E-mail: janaina.moreno@ymail.com. ORCID: http://orcid.org/0000-0002-9806-6352 I'Enfermeira. Mestre. Universidade Federal do Rio de Janeiro. Rio de Janeiro. Brasil. E-mail: mahtob23@gmail.com. ORCID: http://orcid.org/0000-0003-1335-7810 ${ }^{\mathrm{IV}}$ Enfermeiro. Discente do Programa de Residência Multiprofissional em Saúde Mental. Universidade Federal do Rio de Janeiro. Rio de Janeiro. Brasil. E-mail: pabloales81@gmail.com. ORCID: http://orcid.org/0000-0002-4350-4829

vEnfermeira. Especialista. Universidade Federal do Rio de Janeiro. Rio de Janeiro. Brasil. E-mail: gloriaantonian@gmail.com. ORCID: http://orcid.org/0000-0002-2225-2861 V'Enfermeira. Doutora. Professora Permanente. Universidade Federal do Rio de Janeiro. Rio de Janeiro, Brasil. E-mail: sheilaguadagnini@gmail.com. ORCID: http://orcid.org/0000-0001-5752-265X 
Os enfermeiros vivem um processo de trabalho árduo - físico e psicologicamente exaustivo - com altas demandas e longas jornadas laborais; trabalham em turnos, com sobrecarga de trabalho motivada pelo insuficiente número de profissionais para atender às unidades superlotadas. Além disso, nesse ambiente, permeado de relações interpessoais complexas com a equipe multiprofissional, há falta de reconhecimento, autonomia e desvalorização da classe; há o convívio diário com o sofrimento, altas exigências de desempenho das equipes e para com a segurança do paciente. Estes e outros fatores desencadeiam o estresse ocupacional e produzem agravos à saúde física e mental desses profissionais $^{2}$.

Entre as principais repercussões associadas ao estresse ocupacional, têm-se o absenteísmo, insatisfação laboral, acidentes de trabalho, diminuição da qualidade de vida, síndrome de Burnout, problemas cardiovasculares, distúrbios psíquicos menores, além do declínio do desempenho do trabalhador, que impacta a qualidade do cuidado ${ }^{3}$.

$\mathrm{Na}$ contemporaneidade, os enfermeiros convivem com jornadas de trabalho extenuantes, com o subemprego ou duplos/múltiplos vínculos, condições de trabalho inadequadas, falta de materiais, dimensionamento inadequado de profissionais, com um processo de trabalho fragmentado, aumento do nível de complexidade dos pacientes/usuários, necessidade de atualização constante - demandas tecnológicas, inexistência e/ou ineficácia de políticas públicas. Esses aspectos expõem os trabalhadores à intensificação e precarização do trabalho, fatores que levam ao adoecimento e podem comprometer, substancialmente, a qualidade dos cuidados prestados. Diante disso, o trabalho se configura como uma teia que traz, ao trabalhador, uma série de implicações, notadamente, o adoecimento. Doenças como fadiga, lombalgias e ferimentos passam a coexistir com doenças psíquicas: ansiedade, depressão, estresse, síndromes de Burnout e do pânico, as quais passam a fazer parte do dia a dia do trabalhador ${ }^{4}$.

A qualidade de vida no trabalho (QVT) está diretamente relacionada à satisfação e ao bem-estar do indivíduo na execução de suas tarefas e é indispensável no que diz respeito à produtividade e à competitividade, fatores sem os quais uma organização não sobreviveria no mercado ${ }^{5}$.

Muitos desafios se apresentam, os quais se constituem em ações essenciais no sentido de fazer frente aos inúmeros impactos que a contemporaneidade impõe aos enfermeiros. Dentre estes, destacam-se fortalecer a participação em organizações de classe visando ao estabelecimento de construções coletivas, que beneficiem a profissão; participar ativamente da formulação e implementação de políticas públicas voltadas à saúde do trabalhador e regulamentação do trabalho ${ }^{4}$. Dessa forma, este estudo tem como objetivo analisar a associação entre danos à saúde do enfermeiro e qualidade de vida no trabalho.

\section{MÉTODO}

Pesquisa quantitativa, descritiva, transversal, realizada em um Hospital Universitário no Rio de Janeiro. Estudo constituído por enfermeiros que atuavam nas unidades assistenciais de baixa, média e alta complexidade e que trabalhavam na Instituição há mais de seis meses. A população compreendia 230 enfermeiros. Solicitado quadro funcional de enfermagem, contendo as informações: total de enfermeiros, lotação, função, tipo de contratação (estatutário, terceirizado, residente), bem como os que estavam ativos, de férias e afastados por licença médica. Realizou-se cálculo amostral com grau de confiança de $95 \%$ e margem de erro de $5 \%$ e dessa forma, chegou-se a uma amostra de 145 enfermeiros (63\% do quadro funcional).

O mesmo cálculo amostral foi aplicado a cada setor e turno de trabalho, chegando-se ao quantitativo $63 \%$ de enfermeiros que deveriam ser entrevistados por setor e turno, divididos igualmente entre serviço diurno e noturno. Após aprovação da coleta de dados pelos Comitês de Ética em Pesquisa, pareceres números 1.634.051 e 1.643.912, a pesquisa foi realizada com enfermeiros estatutários federais e subordinados a outros tipos de contrato de trabalho. Foram excluídos do estudo enfermeiros em programa de residência de enfermagem.

Para a coleta de dados, utilizou-se um questionário multidimensional que incluía os seguintes módulos: I) características sociodemográficas; II) mensuração dos danos à saúde através de um instrumento adaptado composto por um módulo de questões que avaliava a autopercepção dos trabalhadores sobre os danos à saúde e relacionados ou não com o ambiente de trabalho. As opções de resposta eram distribuídas da seguinte forma: não apresenta o dano; dano provocado pelo trabalho; dano agravado pelo trabalho; e dano sem relação com o trabalho6; III) Para a mensuração de qualidade de vida no trabalho, utilizou-se o Instrumento de Qualidade de Vida no Trabalho de Enfermeiros (IQVTE), versão reduzida e validada ${ }^{7}$.

No instrumento, o conceito de qualidade de vida no trabalho é expresso pela percepção de satisfação dos enfermeiros com aspectos do trabalho considerados importantes. A medida da qualidade de vida no trabalho baseia-se nos graus de satisfação e de importância percebidos pelos enfermeiros em relação a diferentes aspectos do seu trabalho em hospitais. $O$ instrumento original compõe-se de dois questionários totalizando 71 itens ${ }^{8}$. 
O primeiro avalia o nível de satisfação do profissional com determinadas situações vivenciadas no trabalho e o segundo, o grau de importância de cada item correspondente da escala de satisfação. As opções de resposta estão distribuídas em uma escala do tipo Likert com escores que variam de 1 (muito insatisfeito/ nada importante) a 5 (muito satisfeito/ muito importante). 0 instrumento inclui ainda uma opção de resposta, com valor 0 , que possibilita assinalar quando a situação retratada no item não se aplica ao respondente. Nesse sentido o IQVTE avalia as dimensões, a saber: valorização e reconhecimento institucional; condições de trabalho, segurança e remuneração; identidade e imagem profissional; e integração com a equipe.

O procedimento para a atribuição dos escores requereu, primeiramente, que a pontuação dos itens de satisfação fosse recodificada com a finalidade de centralizar o zero na escala. Essa recodificação foi feita subtraindo-se o valor 3 (três) dos escores atribuídos a cada um dos cinco níveis de satisfação, resultando em pontuações de $-2,-1,0,+1$ e +2 , para as pontuações iniciais de 1, 2, 3, 4 e 5, respectivamente; em seguida, os escores recodificados de cada item de satisfação (de -2 a +2 ) foram multiplicados pelos valores brutos dos escores atribuídos aos respectivos itens de importância (de 1 a 5).

O escore total foi obtido somando-se os valores ponderados de todos os itens respondidos e dividindo-se pelo total de itens respondidos. Até esse ponto, os escores podiam variar de $-10 \mathrm{a}+10$. Para eliminar pontuações negativas no escore final, somou-se 10 aos valores obtidos, resultando em uma variação possível de 0 a 20. Os maiores valores indicavam melhor qualidade de vida no trabalho do enfermeiro. Resumidamente, o escore total e para cada dimensão foi calculado com base no algoritmo valor recodificado de cada item de satisfação (SAT) vezes valor bruto de cada item de importância (IMP) [(SAT x IMP) de cada item $\div$ número de itens respondidos] + 10, sendo SAT = valor recodificado de cada item de satisfação $(-2$ a +2$)$ e IMP = valor bruto de cada item de importância ${ }^{7}$. Esse procedimento gerou uma variável contínua que foi categorizada com base nos valores da mediana da distribuição. A confiabilidade do IQVTE foi avaliada através do coeficiente Alpha de Cronbach ${ }^{9}$. Para avaliação do nível de estabilidade das respostas adotaram-se critérios sugeridos por Landis \& Koch (1977): abaixo de zero = pobre; 0 a 0,20 = fraca; 0,21 a 0,40= provável; 0,41 a 0,60 = moderada; 0,61 a 0,80 = substancial e 0,81 a 1,00 = quase perfeita.

No IQVTE, o resultado de valorização e reconhecimento institucional apresentou média de 10 pontos (DP \pm 3 ). Condições de trabalho, segurança e remuneração apresentaram média de 7,5 (DP士 2,9). Identidade e imagem profissional apresentaram média de 11,6 (DP $\pm 3,0)$. Integração com a equipe apresentou média de 12,6 (DP $\pm 3,5)$. Os valores do Alpha de Cronbach encontrados demonstraram que os fatores "condições de trabalho, segurança e remuneração", "identidade e imagem profissional" e "integração com a equipe" apresentam consistência interna substancial (entre 0,61-0,80), já o fator "valorização e reconhecimento institucional" teve consistência interna quase perfeita $^{10}$

Na Figura 1, apresentam-se as dimensões e os pontos de corte adotados, em função da mediana, para a classificação dos grupos de estudo em relação à qualidade de vida no trabalho na utilização do IQVTE.

\begin{tabular}{|c|c|c|c|c|c|c|}
\hline \multirow{2}{*}{ Dimensões IQVTE } & \multirow{2}{*}{$\begin{array}{l}\text { No de } \\
\text { itens }\end{array}$} & \multirow{2}{*}{$\begin{array}{c}\text { Variação } \\
\text { dos escores }\end{array}$} & \multirow{2}{*}{$\begin{array}{l}\text { Média } \\
\text { (DP) }\end{array}$} & \multirow{2}{*}{$\begin{array}{l}\text { Alpha de } \\
\text { Cronbach }\end{array}$} & \multicolumn{2}{|c|}{ Pontos de corte } \\
\hline & & & & & Alta QVT & Baixa QVT \\
\hline Valorização e reconhecimento institucional & 12 & $1,08-15,0$ & $10,0(3,0)$ & 0,887 & Escores $>10,0$ & Escores $\leq 10,0$ \\
\hline Condições de trabalho, segurança e remuneração & 10 & $0,0-16,8$ & $7,5(2,9)$ & 0,801 & Escores $>7,6$ & Escores $\leq 7,6$ \\
\hline Identidade e imagem profissional & 5 & $2,5-18,0$ & $11,6(3,0)$ & 0,792 & Escores $>11,5$ & Escores $\leq 11,5$ \\
\hline Integração com a equipe & 4 & $1,4-19,6$ & $12,6(3,5)$ & 0,624 & Escores $>13,0$ & Escores $\leq 13,0$ \\
\hline Escala global IQVTE & 31 & 1,71-14,61 & $9,8(2,5)$ & 0,924 & & \\
\hline
\end{tabular}

FIGURA 1: Dimensões e pontos de corte adotados para a classificação dos grupos com alta e baixa qualidade de vida no trabalho do enfermeiro, segundo o IQVTE. Rio de Janeiro, RJ, Brasil, 2018.

Os enfermeiros foram entrevistados pela pesquisadora e mais três auxiliares de pesquisa treinados para a aplicação do questionário mediante obtenção da assinatura do Termo de Consentimento Livre e Esclarecido (TCLE). Os questionários eram autopreenchidos.

A caracterização da amostra em relação às variáveis sociodemográficas e relativas aos danos à saúde baseou-se em estatísticas descritivas - média, desvio padrão, assim como valores brutos e percentuais. As análises bivariadas utilizaram por base o teste do qui-quadrado de Pearson e o cálculo da razão de chance e respectivos intervalos de confiança.

Sabe-se que nos estudos de corte transversal os modelos de regressão que estimam a razão de prevalências (RP) mostram-se muito eficientes para estimar medidas pontuais não enviesadas. Contudo, é sabido que a RP tende a ser superestimada nas investigações cujo desfecho tenha alta prevalência no grupo estudado. Desta forma, optou-se por utilizar 
a razão de chance, ou odds-ratio, como método de análise neste estudo de desfechos associados à saúde. Em função do tamanho populacional, não foi possível proceder com análises multivariadas - como é o caso da regressão logística.

Os resultados obtidos na regressão logística perderiam a precisão e comprometeriam a interpretação dos dados. Para o processamento dos dados quantitativos, utilizou-se o programa Statistical Package for the Social Sciences (SPSS), versão 23.

A pesquisa foi desenvolvida respeitando as diretrizes da Resolução número 466/2012 do Conselho Nacional de Saúde, que trata de pesquisa envolvendo seres humanos ${ }^{11}$.

\section{RESULTADOS}

Amostra composta por 145 enfermeiros, com idade média de 44 anos (desvio padrão - DP $\pm 10,8$ ), 82\% do gênero feminino, $57,3 \%$ casados e $96,6 \%$ com pós-graduação, sendo lato sensu $(69,4 \%)$ e stricto sensu $(27,6 \%)$. Com relação às variáveis ocupacionais, a média de tempo de trabalho no hospital foi de 15 anos (DP $\pm 12,1)$. Média de jornada de trabalho semanal em horas foi de 45,2 horas (DP $\pm 14,5)$. Dos participantes, $51 \%$ trabalham em mais de uma instituição, $86,2 \%$ eram servidores públicos federais, $54,8 \%$ estavam lotados no serviço diurno e $75,9 \%$ cumpriam carga horária de 30 horas semanais. Quanto à faixa salarial em salário mínimo (SM) federal, 41,4\% recebiam entre 6 e 8 SM. Em relação ao setor de lotação, a maioria da amostra (63,4\%) estava lotada em setores de baixa complexidade e $36,6 \%$ divididos entre os de média e alta complexidade.

Na Tabela 1, serão apresentados os problemas de saúde autoreferidos pelos enfermeiros. Para efeito da descrição dos resultados, as variáveis relacionadas aos danos à saúde foram categorizadas em três níveis de resposta: "não apresenta", "provocado/agravado pelo trabalho" e "não relacionado ao trabalho".

TABELA 1: Caracterização do grupo estudado em função dos danos à saúde autorreferidos pelos enfermeiros e a percepção de sua relação com o trabalho ( $n=145)$. Rio de Janeiro, RJ, Brasil, 2018.

\begin{tabular}{|c|c|c|c|}
\hline \multirow[b]{2}{*}{ Doenças e sintomas investigados } & \multicolumn{3}{|c|}{ Danos à saúde autorreferidos } \\
\hline & $\begin{array}{c}\text { Não } \\
\text { apresenta } \\
\text { n(\%) }\end{array}$ & $\begin{array}{c}\text { Provocado/ } \\
\text { agravado pelo } \\
\text { trabalho } \\
\mathrm{n}(\%)\end{array}$ & $\begin{array}{c}\text { Não } \\
\text { relacionado } \\
\text { ao trabalho } \\
\text { n(\%) }\end{array}$ \\
\hline Lesões por acidentes & $96(66,2)$ & $46(31,7)$ & $3(2,1)$ \\
\hline Doenças infecciosas & $105(72,4)$ & $37(25,5)$ & $3(2,1)$ \\
\hline Consumo frequente de medicamentos & $95(65,5)$ & $44(30,3)$ & $4(4,1)$ \\
\hline Problemas do sistema nervoso & $89(61,4)$ & $54(37,2)$ & $2(1,4)$ \\
\hline Estresse & $22(15,2)$ & $120(82,8)$ & $3(2,1)$ \\
\hline Depressão & $100(69,0)$ & $41(28,3)$ & $4(2,8)$ \\
\hline Agressões ou condutas violentas & $104(71.7)$ & $38(26.2)$ & $3(2,1)$ \\
\hline Transtornos do sono & $57(39,3)$ & $83(57,2)$ & $5(3,4)$ \\
\hline Mudanças de humor/alterações de comportamento & $56(38,6)$ & $84(57,9)$ & $5(3,4)$ \\
\hline Alcoolismo e uso de outras drogas & $137(94,5)$ & $8(5,5)$ & - \\
\hline Dor de cabeça frequente & $71(49,0)$ & $63(43,4)$ & $11(7,6)$ \\
\hline Perda auditiva & $12(82,8)$ & $16(11,0)$ & $9(6,2)$ \\
\hline Problemas oculares & $84(57,8)$ & $36(4,8)$ & $25(17,2)$ \\
\hline Doenças do coração & $125(86,2)$ & $14(9,7)$ & $6(4,1)$ \\
\hline Hipertensão & $97(66,9)$ & $39(26,9)$ & $9(6,2)$ \\
\hline Varizes & $46(31,7)$ & $92(63,4)$ & $7(4,8)$ \\
\hline Doenças renais & $130(89.7)$ & $10(6,9)$ & $5(3,4)$ \\
\hline Problemas respiratórios & $107(73,8)$ & $32(22,1)$ & $6(4,1)$ \\
\hline Doenças de pele & $115(79,3)$ & $28(19,3)$ & $2(1,4)$ \\
\hline Problemas digestivos & $98(67,6)$ & $42(29,0)$ & $5(3,4)$ \\
\hline Doenças do fígado & $129(89,0)$ & $12(8,3)$ & $4(2,8)$ \\
\hline Fadiga muscular & $63(43,4)$ & $80(55,2)$ & $2(1,4)$ \\
\hline Problemas de articulação & $77(53,1)$ & $67(46,2)$ & $1(0,7)$ \\
\hline Lesões da coluna vertebral & $72(49,7)$ & $70(48,3)$ & $3(2,1)$ \\
\hline Lombalgia & $44(30,3)$ & $98(67,6)$ & $3(2,1)$ \\
\hline Intoxicação por metais ou substâncias químicas & $132(91)$ & $13(9)$ & - \\
\hline Câncer & $137(94,5)$ & $5(3,4)$ & $3(2,1)$ \\
\hline Transtornos relacionados ao ciclo menstrual & $116(80,0)$ & $20(13,8)$ & $9(6,2)$ \\
\hline Transtornos da gravidez ou de órgão reprodutor & $131(90,3)$ & $12(8,3)$ & $2(1,4)$ \\
\hline Afastamentos frequentes por motivo de doença & $113(77,9)$ & $31(21,4)$ & $1(0,7)$ \\
\hline Mudança/transferência do trabalho por motivo de doença & $120(82,8)$ & $24(16,6)$ & $1(0,7)$ \\
\hline
\end{tabular}


No que se refere aos danos à saúde informados pelos participantes que foram provocados e/ou agravados pelas atividades laborais, destacaram-se $(30,3 \%)$ consumo frequente de medicamentos; (37,2\%) problemas do sistema nervoso; (82,8\%) estresse crônico, $(28,3 \%)$ agressões ou condutas violentas; $(57,9 \%)$ mudanças de humor/alterações de comportamento; $(57,2 \%)$ transtornos do sono; $(43,4 \%)$ cefaleia frequente; $(63,4 \%)$ varizes, $(55,2 \%)$ fadiga muscular; $(46,2 \%)$ problemas de articulação; (48,3\%) lesões da coluna vertebral; $(67,6 \%)$ lombalgia; $(21,4 \%)$ afastamentos frequentes por motivo de doença.

Na Tabela 2 são apresentadas as associações entre danos à saúde autorreferidos pelos enfermeiros relacionados ao trabalho e a qualidade de vida no trabalho do enfermeiro.

TABELA 2: Associação entre danos à saúde autorreferidos relacionados ao trabalho e a qualidade de vida no trabalho do enfermeiro expressa com base na razão de chance (RC) e respectivos intervalos de confiança (IC95\%) e no teste do qui-quadrado $n=145$ ). Rio de Janeiro, RJ, 2018

\begin{tabular}{|c|c|c|c|}
\hline \multirow{2}{*}{ Danos à saúde } & \multicolumn{3}{|c|}{ Baixa Qualidade de Vida no Trabalho } \\
\hline & n (\%) & RC (IC95\%) & $\mathbf{p}$ \\
\hline \multicolumn{4}{|l|}{ Lesões por acidentes } \\
\hline Não relatado & $42(44,7)$ & 1,0 & \multirow{2}{*}{0,076} \\
\hline Sim, provocado/agravado & $29(60,4)$ & $1,89(0,93-3,83)$ & \\
\hline \multicolumn{4}{|c|}{ Problemas do sistema nervoso } \\
\hline Não relatado & $40(49,0)$ & 1,0 & \multirow{2}{*}{0,228} \\
\hline Sim, provocado/agravado & $31(56,4)$ & $1,52(0,77-2,99)$ & \\
\hline \multicolumn{4}{|l|}{ Consumo de medicamentos } \\
\hline Não relatado & $42(44,7)$ & 1,0 & \multirow{2}{*}{0,026} \\
\hline Sim, provocado/agravado & $28(65,1)$ & $2,31(1,10-4,88)$ & \\
\hline \multicolumn{4}{|l|}{ Estresse } \\
\hline Não relatado & $10(45,5)$ & 1,0 & \multirow{2}{*}{0,643} \\
\hline Sim, provocado/agravado & $61(50,8)$ & $1,24(0,50-3,09)$ & \\
\hline \multicolumn{4}{|l|}{ Transtornos do sono } \\
\hline Não relatado & $19(33,3)$ & 1,0 & \multirow{2}{*}{0,001} \\
\hline Sim, provocado/agravado & $52(61,2)$ & $3,15(1,56-6,36)$ & \\
\hline \multicolumn{4}{|l|}{ Mudanças de humor } \\
\hline Não relatado & $24(43,6)$ & 1,0 & \multirow{2}{*}{0,228} \\
\hline Sim, provocado/agravado & $47(54,0)$ & $1,52(0,77-2,99)$ & \\
\hline \multicolumn{4}{|l|}{ Depressão } \\
\hline Não relatado & $45(45,9)$ & 1,0 & \multirow{2}{*}{0,077} \\
\hline Sim, provocado/agravado & $25(62,5)$ & $1,96(0,924,17)$ & \\
\hline \multicolumn{4}{|l|}{ Dor de cabeça frequente } \\
\hline Não relatado & $42(58,3)$ & 1,0 & \multirow{2}{*}{0,044} \\
\hline Sim, provocado/agravado & $29(41,4)$ & $1,98(1,02-2,86)$ & \\
\hline \multicolumn{4}{|l|}{ Varizes } \\
\hline Não relatado & $23(50,0)$ & 1,0 & \multirow{2}{*}{0,999} \\
\hline Sim, provocado/agravado & $45(50,0)$ & $1,00(0,50-2,02)$ & \\
\hline \multicolumn{4}{|l|}{ Fadiga muscular } \\
\hline Não relatado & $40(51,3)$ & 1,0 & \multirow{2}{*}{0,880} \\
\hline Sim, provocado/agravado & $31(50,0)$ & $1,05(0,54-2,05)$ & \\
\hline \multicolumn{4}{|l|}{ Problemas de articulação } \\
\hline Não relatado & $33(50,8)$ & 1,0 & \multirow{2}{*}{0,927} \\
\hline Sim, provocado/agravado & $38(50,0)$ & $1,03(0,53-2,00)$ & \\
\hline \multicolumn{4}{|l|}{ Lesões na coluna vertebral } \\
\hline Não relatado & $39(54,9)$ & 1,0 & \multirow{2}{*}{0,240} \\
\hline Sim, provocado/agravado & $32(45,1)$ & $0,67(0,35-1,30)$ & \\
\hline \multicolumn{4}{|l|}{ Lombalgia } \\
\hline Não relatado & $22(50,0)$ & 1,0 & \\
\hline Sim, provocado/agravado & $49(51,6)$ & $1,07(0,52-2,18)$ & 362 \\
\hline
\end{tabular}

Para os resultados na Tabela 2, utilizou-se a frequência de danos que apareciam em mais de $30 \%$ das respostas, bem como os danos que mais acometiam os enfermeiros conforme a literatura corrobora. Na associação entre os danos à saúde autorreferidos relacionados ao trabalho e a qualidade de vida no trabalho do enfermeiro, percebeu-se que o 
consumo de medicamentos provocado/agravado pelo trabalho aumenta em 2,31 vezes a chance do enfermeiro de ter baixa qualidade de vida no trabalho. Em relação aos transtornos do sono provocados/agravados pelo trabalho, estes aumentam em 3,15 vezes a chance de ter baixa qualidade de vida no trabalho e cefaleia frequente provocada/agravada pelo trabalho aumenta em 1,98 vezes a chance de ter baixa qualidade de vida no trabalho.

\section{DISCUSSÃo}

O trabalho hospitalar e o da enfermagem em específico têm sofrido grande influência da política neoliberal e globalizada, levando à precarização das condições e das relações de trabalho, prejudiciais resultados para esse cenário e para a qualidade da assistência prestada. Assim, os profissionais devem entender melhor o processo pelo qual estão passando para se instrumentalizarem e reivindicarem melhores condições de trabalho e de saúde ${ }^{12}$.

Ressalta-se que na instituição onde ocorreu o estudo os problemas estruturais culminaram em uma crise sem precedentes. O hospital é vinculado ao Ministério da Educação e Saúde e presta serviços ao Sistema Único de Saúde (SUS). No entanto, as verbas destinadas ao hospital estão defasadas e as dívidas acumuladas, o que acarretou condições precárias de funcionamento da unidade.

A enfermagem, como qualquer outra profissão da área da saúde, envolve vários fatores de risco, sendo estes agravados no trabalho desenvolvido em instituições hospitalares, uma vez que tais locais são caracterizados como insalubres. O reconhecimento dos riscos no trabalho envolve vários procedimentos para identificação dos fatores e/ou condições/situações que oferecem potencial de dano e, assim, avaliar o risco "significa estimar a probabilidade e a gravidade de que o dano ocorra. Denota-se ainda que nas instituições de saúde, pelo desconhecimento ou por não identificar algumas situações de risco, o trabalhador desenvolve ações sem a devida proteção, podendo favorecer a ocorrência de acidentes laborais, doenças ocupacionais ou agravos à saúde ${ }^{13}$.

Os resultados deste estudo corroboram com o que a literatura demonstra sobre os desgastes físicos e emocionais que as pessoas podem sofrer no ambiente e relações de trabalho implicando no surgimento de doenças, em que os comportamentos adaptativos psicológicos como mecanismo de enfrentamento ficam debilitados ${ }^{14}$.

O ambiente físico e social do trabalho hospitalar é tido como penoso, perigoso e insalubre e o perfil de adoecimento dos trabalhadores que nele executam suas atividades ainda é pouco conhecido pelos gerentes institucionais e órgãos da esfera pública. Também há muito que se pesquisar acerca dos problemas de saúde decorrentes da sobrecarga de trabalho, do esforço exigido na realização das atividades e das condições de trabalho inadequadas que geram encargos financeiros pagos pela previdência social. Nesse sentido, o fato de o Estado e seus cidadãos arcarem com um ônus que é das instituições, que propiciam o adoecimento dos seus trabalhadores, é, sem dúvida, uma questão ética e política a ser discutida ${ }^{15}$.

No presente estudo, evidenciou-se que o consumo de medicamentos aumenta em 2,31 a chance de qualidade de vida ruim do enfermeiro e os transtornos do sono aumentam essa chance em 3,15 vezes. $O$ trabalho por turnos e o trabalho noturno são estressores laborais, considerando que a jornada noturna propicia déficit de sono e invariavelmente os profissionais recorrem ao consumo de medicamentos para se manter acordados e/ou para poder descansar, considerando as jornadas duplas e triplas de trabalho. Ressalta-se que a falta de sono também provoca problemas de vigilância que podem incorrer em prejuízos para a assistência ao paciente, fadiga, transtornos de humor, assim como isolamento social, problemas familiares e sociais.

Para obter conforto diante de perturbações de ordem física ou psíquica, enfermeiros recorrem ao uso de terapias medicamentosas, principalmente utilizando-se da automedicação. Em particular, o consumo de substâncias psicoativas faz parte do cotidiano dos trabalhadores de enfermagem. Segundo o Observatório Brasileiro de Informações Sobre Drogas (OBID), os medicamentos psicoativos (também denominados psicotrópicos) são substâncias capazes de atuar no sistema nervoso central, deprimindo-o (a exemplo dos álcoois, barbitúricos, benzodiazepínicos e opiáceos), estimulando-o (como as anfetaminas, a cocaína e o tabaco) ou perturbando-o (como a maconha, alucinógenos, anticolinérgicos). Além do uso terapêutico, essas substâncias costumam ser utilizadas para o aumento da sensação de bem-estar, sem prescrição médica. Diante do exposto, deve-se atentar para os efeitos resultantes do uso dessas substâncias que podem envolver alterações na mente, no corpo e na conduta, o que traz riscos tanto para as pessoas que as utilizam quanto para os que com elas convivem, podendo interferir, diretamente, na saúde e qualidade de vida dos trabalhadores ${ }^{16}$.

Os distúrbios osteomusculares relacionados ao trabalho - DORT abrangem um grupo heterogêneo de agravos do sistema musculoesquelético causados por processo crônico, desenvolvido por atividades realizadas durante a atividade laboral. Essas atividades acometem músculos, tendões, articulações, nervos e ligamentos, apresentando variado quadro clínico, incluindo queixas de dor, formigamento dormência, peso e fadiga precoce. É um dos problemas mais frequentes e mais dispendiosos entre profissionais de enfermagem, contribuindo significativamente para a incapacidade e o 
afastamento do trabalho. Dentre as profissões da área da saúde, a enfermagem tem sido a mais afetada, uma vez que tais trabalhadores desenvolvem as atividades em diversos locais, dentre estes as instituições hospitalares, realizando atividades de forma contínua, que exigem atenção constante e esforço físico. É um trabalho repetitivo, que demanda esforço físico, levantamento de peso (pacientes com sobrepeso ou obesidade), posturas inadequadas, que são exigidas durante a prestação de cuidados, e a falta de adequação arquitetônica dos locais de trabalho, os quais associados aos estressores mentais são fatores de risco para ocorrência desses distúrbios. Os distúrbios osteomusculares relacionados ao trabalho não são causados por um esforço repetitivo qualquer. Suas causas vão além dos sintomas físicos, pois elas passam pela organização do trabalho, dificuldades interpessoais, bem como os fatores ergonômicos intrínsecos ao ambiente laboral ${ }^{17}$.

Este trabalho evidenciou que cefaleia frequente provocada/agravada pelo trabalho aumenta em 1,98 vezes a chance de o enfermeiro ter baixa qualidade de vida no trabalho. Segundo a Organização Mundial de Saúde (OMS), as cefaleias são queixas de relevância na população, tornando-as, muitas vezes, incapazes de suas ocupações diárias. Muitos dos indivíduos que sofrem cefaleia menos intensas não procuram assistência médica e os que sofrem de cefaleias ou crises frequentes nem sempre têm acesso aos serviços de saúde, tornando-se problema não solucionado diante o sistema de saúde. Um estudo identificou a frequência de cefaleia em funcionários dos hospitais de uma cidade da região oeste II do estado de Goiás e demonstrou que os profissionais que mais apresentavam cefaleia eram os enfermeiros (27\%), e a frequência de cefaleia entre os enfermeiros foi de $83,3 \%$. Uma explicação para esse episódio foi que esses trabalhadores estavam expostos ao estresse contínuo, com carga horária excessiva de trabalho, acarretando poucas horas de sono ${ }^{18}$.

As condições oferecidas, para que o trabalho na área da saúde seja desenvolvido, contribuem para a "qualidade do serviço e o desempenho dos profissionais. Além disso, as sobrecargas físicas e mentais desses trabalhadores se relacionam. Atenção, segurança na execução de técnicas e cuidados constantes podem gerar o desgaste psíquicoemocional dos profissionais e, levar, consequentemente, a agravos e doenças ocupacionais ${ }^{13}$.

A implantação de medidas preventivas no ambiente de trabalho e a educação permanente seriam boas estratégias para minimizar o desenvolvimento e as consequências desses distúrbios e, consequentemente, reduzir os afastamentos e as licenças desses profissionais. O estímulo à prática de exercícios regulares e o manejo dos fatores de risco por meio de intervenções ergonômicas também seriam ações que poderiam auxiliar na melhora dos sintomas ${ }^{17}$.

\section{Limitações do estudo}

Os limites do estudo estão relacionados ao fato da pesquisa ter sido realizada com uma amostra específica e em uma única instituição de saúde pública federal. Dessa maneira, estudos de qualidade de vida no trabalho do enfermeiro devem ser incentivados nos diversos cenários assistenciais e acadêmicos.

\section{CONCLUSÃO}

A ocorrência de danos à saúde do enfermeiro merece profunda discussão considerando sua gravidade e repercussão negativa na qualidade de vida do trabalho do enfermeiro. Ambientes não saudáveis de trabalho favorecem o adoecimento mental e físico desses profissionais. A qualidade de vida no trabalho do enfermeiro é avaliada mediante a satisfação das necessidades pessoais e profissionais. 0 impacto das condições de trabalho na qualidade de vida do trabalho enfermeiro merece atenção, pois é fato que ambientes adequados de trabalho proporcionam satisfação pessoal e profissional, além de manterem a excelência da força de trabalho do enfermeiro.

Como contribuições este estudo permite o avanço do conhecimento científico por meio da identificação dos danos à saúde dos enfermeiros relacionados à sua prática laboral e como esses danos podem interferir negativamente na qualidade de vida no trabalho desses profissionais.

O levantamento desses dados pode propiciar o planejamento de ações que contribuam para ambientes de trabalho mais saudáveis, incentivando uma nova prática no cotidiano de trabalho do enfermeiro na organização hospitalar, bem como em outras organizações de saúde e ensino.

\section{REFERÊNCIAS}

1. Cossi MS, Costa RRO, Medeiros SM, Menezes RMP. The ability to work of the nursing staff inserted in the hospital environment. Rev. Aten. Saúde [Internet]. 2015 [cited 2019 Jan 25]; 13(43):68-72. DOI: https://doi.org/10.13037/rbcs.vol13n43.2676

2. Sarafis $P$, Rousaki E, Tsounis A, Malliarou M, Lahana L, Bamidis $P$, et al. The impact of occupational stress on nurses' caring behaviors and their health related quality of life. BMC Nurs [Internet]. 2016 [cited 2019 Jan 25]; 15:56. DOI: https://doi.org/10.1186/s12912-016-0178-y

3. Scholze AR, Martins JT, Robazzi MLCC, Haddad MCFL, Galdino MJQ, Ribeiro RP. Occupational stress and associated factors among nurses at public hospitals. Cogitare enferm. [Internet]. 2017 [cited 2019 Jan 25]; 22(3). DOI: http://dx.doi.org/10.5380/ce.v22i3.50238 
4. Camponogara S. Desafios do trabalho da enfermagem na contemporaneidade. Rev. espaçciênc. saúde [Internet]. 2017 [cited 2019 Jan 25]; 5(2). Available from: http://revistaeletronica.unicruz.edu.br/index.php/enfermagem/article/view/6758/1320

5. Amaral JF, Ribeiro JP, Paixão DX. Quality of life at work of nursing professionals in hospitals: an integrated review. Espaç. saúde (Online). 2015 [cited 2019 Jan 25]; 16(1):66-74. Available from: http://espacoparasaude.fpp.edu.br/index.php/espacosaude/article/view/419/pdf_64

6. Boix P, Vogel L. La evolucíon de riesgosem los lugares de trabajo: guia para umainvestigacíonsindical. Bruxelles: BTS, 1997.

7. Kimura M, Carandina DM. Development and validation of a short form instrument for the evaluation of quality of working life of nurses in hospitals. Rev. Esc. Enferm. USP [Internet]. 2009 [cited 2019 Jan 25]; 43(spe):1044-54. Available from: http://www.scielo.br/pdf/reeusp/v43nspe/en_a08v43ns.pdf

8. Carandina DM. Qualidade de vida no trabalho: construção e validação de um instrumento de medida para enfermeiras [Doctoral dissertation]. São Paulo: Universidade de São Paulo; 2003.

9. Norman GR, Streiner DL. Biostatistics: the bare essentials. Canada: Pmph USA, 1998.

10. Landis JR, Koch GG. The measurement of observer agreement for categorical data. Biometrics [Internet]. 1977 [cited 2019 Jan 25]; 33(1):159-74. DOI: https://doi.org/10.2307/2529310

11. Conselho Nacional de Saúde (Br). Resolução no 466, de 12 de dezembro de 2012. Aprova diretrizes e normas regulamentadoras de pesquisas envolvendo seres humanos [Internet]. Brasília (DF): Ministério da Saúde; 2012 [cited 2019 Jan 25]. Available from: https://bvsms.saude.gov.br/bvs/saudelegis/cns/2013/res0466_12_12_2012.html

12. Gonçalves FGA, Souza NVDO, Zeitoune RCG, Adame GFPL, Nascimento SMP. Impacts of neoliberalism on hospital nursing work. Texto\&contexto enferm. [Internet]. 2015 [cited 2019 Jan 25]; 24(3):646-53. DOI: http://dx.doi.org/10.1590/010407072015000420014

13. Martins JT, Bobroff MCC, Andrade AN, Menezes GD. Emergency nursing team: occupational risks and self protection. Rev. enferm. UERJ [Internet]. 2014 [cited 2019 Jan 25]; 22(3):334-40. Available from: https://www.epublicacoes.uerj.br/index.php/enfermagemuerj/article/view/13690/10481

14. Silva JLL, Soares RS, Costa FS, Ramos DS, Lima FB, Teixeira LR. Psychosocial factors and prevalence of burnout syndrome among nursing workers in intensive care units. Rev. bras. ter. intensiva [Internet]. 2015 [cited 2019 Jan 25]; 27(2):125-33. DOI: https://doi.org/10.5935/0103-507X.20150023

15. Fabri JMG, Noronha IR, Oliveira EB, Kestenberg CCF, Harbache LMA, Noronha IR. Occupational stress in pediatric nurses: physical and psychological manifestations. Rev. baiana enferm. [Internet]. 2018 [cited 2019 Jan 25]; 32:e25070. DOI: https://doi.org/10.18471/rbe.v32.25070

16. Vieira TG, Beck CLC, Dissen CM, Camponogara S, Gobatto M, Coelho APF. Illness and the use of psychoactive drugs among nursing workers at intensive care units. Rev. enferm. UFSM [Internet]. 2013 [cited 2019 Jan 25]; 3(2):205-14. Available from: https://periodicos.ufsm.br/reufsm/article/view/7538/pdf

17. Oliveira VC, Almeida RJ. Aspects that determine the musculoskeletal disorders in nursing professionals and their psychosocial impact. J. health sci. [Internet]. 2017 [cited 2019 Jan 25]; 19(2):130-5. DOI: https://doi.org/10.17921/24478938.2017v19n2p130-135

18. Pinto JMM, Ferreira MM, Costa MB, Garcia SAS, Andrade WM, Fernandes CKC, et al. Frequency of headache in employees of hospitals in a city on the west region II of Goiás state. Rev. FMB [Internet]. 2015 [cited 2019 Jan 25]; 8(1):1-15. Available from: http://scholar.googleusercontent.com/scholar?q=cache:5dUo8iO_rHoJ:scholar.google.com/\&hl=pt-BR\&as_sdt=0,5 